\title{
Efecto analgésico del bloqueo PENG (grupo de nervios pericapsulares) en pacientes con fractura de cadera
}

\author{
Analgesic effect of the PENG lock (pericapsular nervous \\ group) in patients with hip fracture
}

Marcelo Molinelli', Jhonathan E. O. Romero', Sebastián Uranga', Josefina Bartolini', Nicolás Caputo', Lamberto Ariel'1, Raúl Torres'1, Claudio Vales

\begin{abstract}
Ultrasound regional blockade emerged that blocks the branches of the femoral nerve, obturator and accessory obturator that innervate the anterior hip capsule, the PENG block (group of pericapsular nerves), which by its Recent description does not have enough evidence in medical practice. Objectives: To verify the analgesic effect of the PENG block in patients with hip fracture and its analgesic permanence during the first 10 hours after the block in patients admitted with a diagnosis of hip fracture, at the General Interzonal Hospital of Acute "Dr Oscar E Alende "From Mar del Plata, Argentina, in the months of May to November 2019. Materials and Methods: A prospective descriptive observational study was carried out with a total of 53 patients, hospitalized patients with a diagnosis of hip fracture, with standardized intravenous analgesic scheme and who have not yet undergone hip surgery. Pain was evaluated with the EVA scale (visual analog scale) prior to the blockage, and then at 30 min and 10 hours after the blockade, $15 \mathrm{ml}$ of $1 \%$ lidocaine and $15 \mathrm{ml}$ of bupivacaine at 0 were used. $25 \%$, convex or linear ultrasound probe according to patient weight and $100 \mathrm{~mm}$ needle. In order to reproduce and evaluate the pain, the patients had a $30^{\circ}$ flexion of the hip. Results: Prior to the blockade, $66 \%$ of the patients had severe pain and $34 \%$ moderate pain, none presented mild pain or absence, both at thirty minutes and ten hours after the blockade, no patient presented severe pain and all patients presented analgesia with a decrease in more than three points on the VAS scale, in some cases reaching a decrease of 10 points on that scale. Conclusions: The PENG block is a regional anesthesia technique that provides very good analgesia to patients with hip fractures, therefore, it is an excellent saving strategy for systemic analgesics. Knowing the analgesia provided by the blockade at $30 \mathrm{~min}$ and at $10 \mathrm{~h}$, it could
\end{abstract}

Key words:

Hip fracture, analgesic effect, PENG block

Hospital Interzonal General de Agudos "Dr Oscar E Alende". Mar del Plata, Argentina.

Fecha de ingreso: 10 de noviembre de 2019

Fecha de recepción: 01 de diciembre de 2019

\section{ORCID}

https://orcid.org/0000-0002-9039-5215

Correspondencia:

Email: drmolinelli@gmail.com 

nelli et al.

be performed both in the preoperative period for the transfer and mobilization of the patient, as well as in the postoperative period, which could save the use of opioids and decrease hospital stay.

\section{RESUMEN}

Introducción: La fractura de cadera es una emergencia ortopédica común en ancianos asociada a gran morbimortalidad, una adecuada analgesia regional perioperatoria determina un ahorro en el uso de analgésicos sistémicos. Recientemente, en el año 2018, surgió un nuevo bloqueo regional ecoguiado muy prometedor que bloquea las ramas del nervio femoral, obturador y obturador accesorio que inervan la capsula anterior de la cadera, el bloqueo PENG (grupo de nervios pericapsulares), el cual por su reciente descripción no cuenta con la suficiente evidencia en la práctica médica. Objetivos: Comprobar el efecto analgésico del bloqueo PENG en pacientes con fractura de cadera y su permanencia analgésica durante las 10 primeras horas posteriores al bloqueo en los pacientes internados con diagnóstico de fractura de cadera, en el Hospital Interzonal General de Agudos "Dr. Oscar E Alende" de Mar del Plata, Argentina, en los meses de mayo a noviembre del 2019. Materiales y Métodos: Se realizó un estudio observacional descriptivo prospectivo con un total de 53 pacientes, se incluyeron pacientes internados con diagnóstico de fractura de cadera, con esquema analgésico endovenoso estandarizado y que aún no hayan sido sometido a cirugía de cadera. Se evaluó el dolor con la escala EVA (escala análoga visual) previo al bloqueo, y luego a los 30 min y a las $10 \mathrm{Hs}$ de haber realizado el bloqueo, para este se utilizaron $15 \mathrm{ml} \mathrm{lidocaína} 1 \%$ y $15 \mathrm{ml}$ de bupivacaína al 0,25\%, sonda ecográfica convexa o lineal según el peso del paciente y aguja $100 \mathrm{~mm}$. Para reproducir y evaluar el dolor se les realizo a los pacientes una flexión de $30^{\circ}$ de la cadera. Resultados: Previo al bloqueo el $66 \%$ de los pacientes tuvieron dolor severo y $34 \%$ dolor moderado, ninguno presentaba dolor leve o ausencia del mismo, tanto a los treinta minutos como a las diez horas posteriores al bloqueo ningún paciente presento dolor severo y todos los pacientes presentaron analgesia con una disminución en más de tres puntos en la escala de EVA, llegando en algunos casos a una disminución de 10 puntos de dicha escala. Conclusiones: El bloqueo PENG es una técnica de anestesia regional que brinda muy buena analgesia a los pacientes con fractura de cadera, por consiguiente, es una excelente estrategia ahorradora de analgésicos sistémicos. Conociendo la analgesia que brinda el bloqueo a los 30 min y a las $10 \mathrm{~h}$ de realizado, se podría realizar dicho bloqueo tanto en el preoperatorio para el traslado y movilización del paciente, como en el post-operatorio, lo que podría ahorrar el uso de opioides y disminuir la estancia hospitalaria.

\section{Palabras clave:}

Fractura de cadera, efecto analgésico, bloqueo PENG

\section{Introducción}

a fractura de cadera es una emergencia ortopédica frecuente en la población anciana, asociada a elevada morbimortalidad[1],[2],[3],[4].

El uso de analgésicos sistémicos en especial los opioides en estos pacientes deteriora en gran medida su recuperación, produciendo mayor incidencia de complicaciones tales como náuseas, vómitos, estados de sedación, constipación, entre otros, llevando a una recuperación y alta más tardías[1],[2], lo que conlleva a una prolongada internación y mayores gastos de recursos hospitalarios.

En el presente trabajo haremos referencia al bloqueo PENG (pericapsular nerve group), el cual fue descripto por primera vez a fines del 2018[6]. Se trata de un bloqueo regional que brinda analgesia en la cadera, principalmente en la zona con más porcentaje 
de inervación sensitiva que corresponde a la cápsula anterior de la articulación[6]. En este bloqueo se ven involucrados el nervio obturador, el obturador accesorio y las ramas proximales del nervio femoral, encargados de la inervación sensitiva en este nivel, con escaso compromiso de la inervación motora, siendo de esta manera el bloqueo PENG aparentemente una mejor opción que los bloqueos tradicionalmente usados para analgesia de cadera como son el bloqueo 3 en 1 , el bloqueo del nervio femoral y el bloqueo de la fascia iliaca, ya que estos últimos no llegan a bloquear el nervio obturador[7],[8],[9], el cual es de gran importante en la inervación de la cadera, en contraste como única desventaja en cuanto a la analgesia del bloqueo PENG, este no llega a bloquear el nervio femorocutáneo lateral (NFCL), el cual es imprescindible abarcar para brindar analgesia en la región cutánea lateral del muslo, zona donde se hacen la mayoría de las incisiones para abordaje quirúrgico de las cirugías de cadera[5].

Al ser un bloqueo de reciente descripción, existe actualmente poca información sobre su efecto analgésico en la práctica clínica[10].

El objetivo propuesto con este estudio es evaluar el efecto analgésico del bloqueo PENG en pacientes con fractura de cadera y comprobar si dicha analgesia se mantiene durante las 10 primeras horas posteriores a su realización, en el Hospital Interzonal General de Agudos "Dr. Oscar E. Alende", Mar del Plata, Argentina.

\section{Materiales y Métodos}

Se realizó un estudio observacional descriptivo prospectivo con un total de 53 pacientes internados entre los meses de mayo a noviembre del año 2019 en el servicio de traumatología del Hospital Interzonal General de Agudos "Dr. Oscar E. Alende" de Mar del Plata, Argentina, con previa autorización del comité de ética de dicho nosocomio y consentimiento informado de cada paciente. Los criterios de inclusión fueron: pacientes que tengan fractura de cadera con dolor y que no hayan sido sometidos a cirugía de cadera ni a una anestesia raquídea o regional durante la internación, para así no sesgar los resultados con la analgesia que produce la reparación de la fractura y la misma anestesia subaracnoidea o regional. Se excluyeron del trabajo los pacientes que no presentaban dolor, los pacientes que tenían una tracción en el miembro fracturado, pacientes no lúcidos o no colaboradores, pacientes con infección en la zona de punción, pacientes con alteraciones en la coagulación, pacientes que se niegan a realizarse el bloqueo PENG, también se excluyeron los pacientes que durante la internación recibieron por algún motivo (alergia medicamentosa, efectos adversos a las drogas, etc.) otro tratamiento analgésico diferente al estandarizado para dicho trabajo de investigación. El plan analgésico estandarizado consiste en $150 \mathrm{mg}$ de diclofenac, y 150 mg de tramadol al día de forma intravenosa en infusión continua.

A todos los pacientes se le evaluó el dolor previo al bloqueo PENG, a los 30 min y a las 10 horas del mismo. Para evaluar el dolor se utilizó una estrategia dinámica, realizándole a los pacientes una flexión pasiva de la cadera de $30^{\circ}$ o menos en caso de alcanzar el máximo dolor y posteriormente el paciente da un puntaje a dicho dolor según la escala visual análoga (EVA)[11], diferenciándolo en leve (0-2), moderado (3 al 7) e intenso (8 al 10).

Para realizar el bloqueo se coloca a los pacientes en posición supina, se usó la sonda de ultrasonido convexa (baja frecuencia) para pacientes de más de $70 \mathrm{~kg}$ y sonda lineal (alta frecuencia) para los pacientes de menos de $70 \mathrm{~kg}$. Con el operador del lado de la cadera comprometida, se coloca la sonda en el plano transversal en la espina iliaca antero inferior, se hace girar la sonda en sentido horario o antihorario (estando la derecha o a la izquierda del paciente respectivamente) aproximadamente $45^{\circ}$ para que se sea alineada con la rama púbica, observándose la eminencia iliopúbica en lo profundo (4 a $6 \mathrm{~cm}$ de profundidad), el musculo psoas apoyado sobre dicha eminencia con su tendón hiperecogénico en lo profundo del músculo en íntima cercanía con la eminencia iliopúbica, más superficial y medial de la imagen obtenida veremos de lateral a medial la arteria y la vena femoral respectivamente. Una vez lograda la imagen ecográfica se introduce la aguja (de $100 \mathrm{~mm}, 22 \mathrm{G}$ ) de lateral a medial. Aunque la técnica ecoguiada adoptada en nuestro trabajo fue en plano, de lateral a medial, vale destacar que introduciendo la aguja con esta técnica se corre el riesgo de una lesión inadvertida del NFCL, y si se realiza el ingreso de medial a lateral se debe tener precaución con la lesión inadvertida del nervio femoral, por lo que ingresar la aguja fuera de plano puede ser una opción segura de no lesionar dichos nervios. La aguja se avanza hasta colocar su punta por debajo y medial al tendón del psoas y por encima de la eminencia iliopúbica, luego se inyectan $15 \mathrm{ml}$ de lidocaína $1 \%$ con epinefrina y $15 \mathrm{ml}$ de bupivacaína al 0,25\% (total 30 $\mathrm{ml}$ de anestésico local), dicho anestésico se deposita entre el tendón del psoas (dispuesto anteriormente) y la eminencia iliopúbica (dispuesta posteriormente) Y se observa como el anestésico baña el musculo psoas 


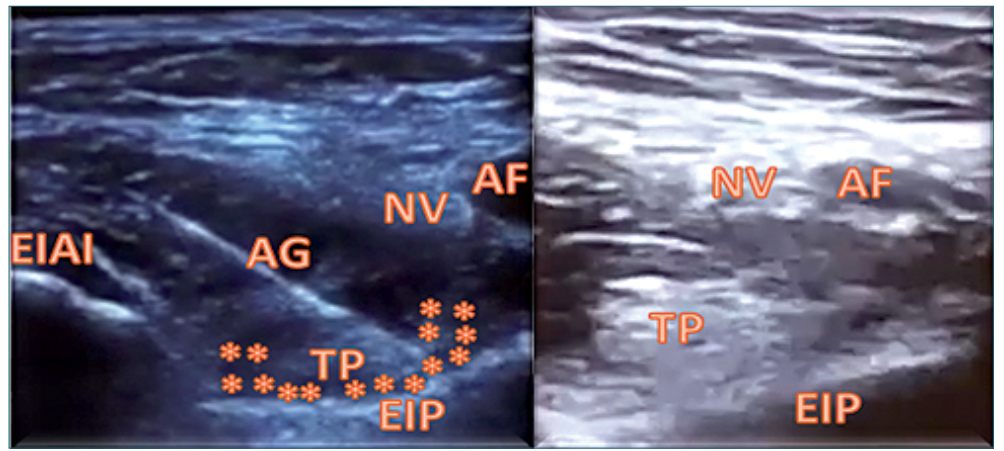

Figura 1. imagen ecogáfica con sonda lineal. AF: arteria femoral, NF: nervio femoral, TP: tendón del psoas, EIAI: espina iliaca antero inferior, Asterisco: anestésico local. EIP: eminencia iliopúbica, AG: aguja.

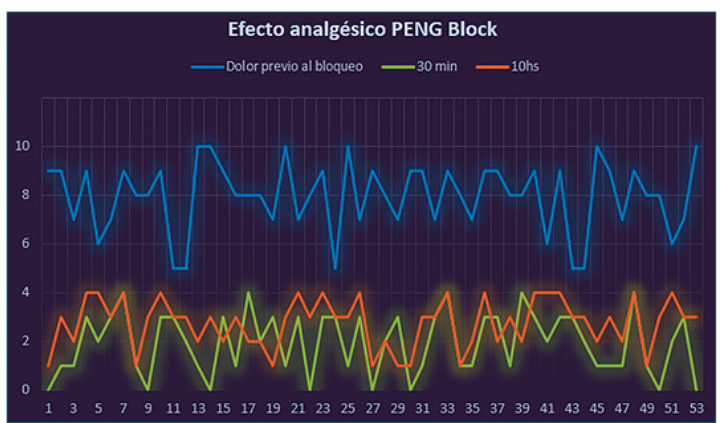

Figura 2. Evaluación del efecto analgésico del bloqueo PENG.

separando el tendón del hueso (eminencia iliopúbica).

\section{Resultados}

Previo al bloqueo PENG, el 34\% de los pacientes presentaban dolor moderado y el otro $66 \%$ dolor severo. Ninguno presentaba dolor leve o ausencia del dolor.

A los 30 min posteriores al bloqueo, ningún paciente presento dolor intenso, 23 pacientes (43\%) presentaron dolor moderado y 30 pacientes $(57 \%)$ presentaron dolor leve o ausencia del mismo.

A las $10 \mathrm{~h}$ de realizado el bloqueo, ninguno presentó dolor severo, 34 pacientes $(64 \%)$ presentaron dolor moderado y 19 pacientes (36\%) dolor leve.

En el total de los casos el bloqueo PENG proporciona analgesia, ya que logro disminuir más de 3 puntos la escala analgésica del dolor (EVA) en todos los pacientes, tanto a los 30 min como a las $10 \mathrm{~h}$ posteriores a la realización de dicho bloqueo, llegando a disminuir en algunos casos hasta 10 puntos la escala del dolor. Aunque no fue el objetivo de nuestra investigación, observamos que ningún paciente refirió más de 4 puntos en la escala EVA durante las 10 primeas horas de realizado el bloqueo, por lo que no fue necesario realizar rescates de morfina ( $2 \mathrm{mg}$ ) a ninguno de los pacientes.

\section{Conclusiones}

Concluimos que el bloqueo PENG es una muy buena estrategia para brindar analgesia en pacientes con fractura de cadera, prolongando su efecto analgésico en por lo menos 10 hrs de realizado el bloqueo, por lo que sería una opción a tener en cuenta tanto para brindar analgesia en el preoperatorio para el traslado y movilización del paciente, como para el post operatorio de la mayoría de las cirugías de cadera. Se podrían hacer futuros estudios que comprueben la eficacia analgésica del bloqueo PENG con infusión continua por catéter, principalmente para los pacientes con fractura de cadera quienes tienen demora para el turno quirúrgico o para quienes tengan contraindicado el uso de analgésicos sistémicos.

La analgesia producida por el bloqueo PENG, conlleva en estos pacientes una marcada disminución del uso de analgésicos sistémicos, sin los efectos adversos de éstos, lo cual podría disminuir la estancia y los costos hospitalarios.

Debido al poco compromiso motor que presenta el bloqueo PENG, podría ser una excelente estrategia para cirugías ambulatorias de cadera, dando la posibilidad de que el paciente tenga una temprana movilidad y disminución de las caídas por debilidad del miembro, por lo que se podrían realizar futuros estudios que evalúen el compromiso motor de este bloqueo. 


\section{Referencias}

1. Titler MG, Herr K, Brooks JM, Xie XJ, Ardery G, Schilling ML, et al. Translating research into practice intervention improves management of acute pain in older hip fracture patients. Health Serv Res. 2009 Feb;44(1):26487. https://doi.org/10.1111/ j.1475-6773.2008.00913.x PMID:19146568

2. Godoy Monzón D, Vazquez J, Jauregui JR, Iserson KV. Pain treatment in post-traumatic hip fracture in the elderly: regional block vs. systemic non-steroidal analgesics. Int J Emerg Med. 2010 Nov;3(4):321-5. https:// doi.org/10.1007/s12245-0100234-4 PMID:21373300

3. World Health Organization. Assessment of fracture risk and its application to screening for osteoporosis. WHO technical report series 843. Geneva: WHO; 1994.

4. Sernbo I, Johnell O. Consequen- ces of a hip fracture: a prospective study over 1 year. Osteoporos Int. 1993 May;3(3):148-53. https://doi.org/10.1007/ BF01623276 PMID:8481591

5. Segado $M$, et al. Eficacia del bloqueo de los nervios obturador y femorocutáneo para analgesia postoperatoria en cirugía de cadera. Rev Esp Anestesiol Reanim. 2009;56(10):590-7. https://doi.org/10.1016/S00349356(09)70474-8.

6. Girón-Arango L, Peng PW, Chin KJ, Brull R, Perlas A. Pericapsular Nerve Group (PENG) block for hip fracture. Reg Anesth Pain Med. 2018 Nov;43(8):859-63. https://doi.org/10.1097/ AAP.0000000000000847 PMID:30063657

7. Marhofer P, Nasel C, Sitzwohl C, Kapral S. Magnetic resonance imaging of the distribution of local anesthetic during the threein-one block. Anesth Analg. 2000 Jan;90(1):119-24. https:// doi.org/10.1097/00000539-

\section{0-00027}

PMID:10624991

8. Swenson JD, Davis JJ, Stream JO, Crim JR, Burks RT, Greis PE. Local anesthetic injection deep to the fascia iliaca at the level of the inguinal ligament: the pattern of distribution and effects on the obturator nerve. J Clin Anesth. 2015 Dec;27(8):652-7. https://doi.org/10.1016/j. jclinane.2015.07.001 PMID:26277873

9. González Castilla R, Bustamante Y, et al. Bloqueo 3 en 1 para analgesia en pacientes con fractura de cadera. Medisan (Santiago De Cuba). 2012;16(11):1698-706.

10. Mistry T, Sonawane KB, Kuppusamy E. PENG block: points to ponder. Reg Anesth Pain Med. 2019 Jan;rapm-2018-100327. PMID:30635514

11. Herrero V, Bueno S, Moyá, et al. Valoración del dolor. Revisión comparativa de escalas y cuestionarios. Rev Soc Esp Dolor. 2018;25(4):228-36. 